\title{
ELEMENTARY SEQUENCES
}

\author{
SCOTT J. BESLIN \\ Department of Mathematics \\ Nicholls State University \\ Thibodaux, LA 70310
}

(Received December 10, 1990 and in revised form September 18, 1991)

ABSTRACT. Explicit finite algebraic formulas are given for sone well-known sequences, including complements of polynomial sequences.

KEY WORDS AND PHRASES. Polynomial sequences, transcendental numbers. 1980 AMS SUBJECT CLASSIFICATION CODES. 11B34, $11 Y 55$.

\section{INTRODUCTION.}

For each integer $n>1$, we may define the function $f_{n}$ on $Z^{+}$, the set of positive integers, by $f_{n}(i)=$ the $\underline{i}$ th positive integer which is not a perfect nth power. For example, the first several terms of the sequence $\left\langle f_{2}(i)\right\rangle$ are $2,3,5,6,7,8,10,11,12,13,14,15,17$. In [1], the formula

$$
f_{2}(i)=i+[\sqrt{i+[\sqrt{i}]}]
$$

is established, where $[x]$ denotes the greatest integer less than or equal to $x$. Similar formulas can be found in [2] and [3]. The authors of [4] extend this result by deriving an explicit formula for $f_{n}$. Indeed, the theorem in [4] gives

$$
f_{n}(i)=i+\left[i^{1 / n}\right]+[i / p(n, i)] \text {, }
$$

$$
\text { where } p(n, i)=\left(\left[i^{1 / n}\right]+1\right)^{n}-\left[i^{1 / n}\right] \text {. }
$$

Furthermore, the authors define a function to be elementary if it has an explicit finite formula involving only the elementary algebraic operations of addition, subtraction, multiplication, division, roots, powers, and greatest integer. A sequence $s=\left\langle s_{j}\right\rangle$ is elementarily generated (e.g) if there exists an elementary function $f$ for which $f(i)=s_{i}, i=1,2,3, \ldots$. If the sequence $s$ is an injective (strictly increasing) sequence of positive integers, the complement of $\underline{s}, \overline{\mathbf{s}}$, is the subsequence of $\langle 1,2,3, \ldots\rangle$ obtained by deleting all terms of $s$. For example, if $s=\left\langle i^{2}\right\rangle=\langle 1,4,9, \ldots\rangle$, then $\bar{s}=\left\langle f_{2}(i)\right\rangle$. A cardinality argument shows that there are uncountably many sequences which are not e.g.

It is the purpose of the present paper to answer some questions raised in 
[4], to broaden the scope of results from [1], [2], [3], and [4], and to address some notions related to e.g. sequences. Several open problems will be posed for readers. We initiate this study by showing that some often-used functions are elementary. Henceforth, we call elementary functions 'e.g.' .

Example 1. The absolute value function is e.g., since the absolute value of $x$ is $\sqrt{x^{2}}$.

Example 2. As a consequence of the division algorithm for integers, the residue function modulo $k, r_{k}: Z^{+} \rightarrow\{0,1, \ldots, k-1\}$ given by $r_{k}(n)=n \bmod k$, is e.g. The expression $n$ mod $k$ denotes the remainder left when $n$ is divided by $k$. This function is elementary because surely

$$
r_{k}(n)=n-[n / k] k \text {. }
$$

For example, $r_{5}(22)=22 \bmod 5=2=22-20=22-[22 / 5](5)$.

Example 3. The very useful Kronecker delta functions

$$
\delta_{k, n}=\left\{\begin{array}{ll}
1 & n=k ; \\
0 & n \neq k
\end{array} \quad \text { ( } k\right. \text { a fixed positive integer) }
$$

are e.g. We leave it to the reader to verify that

$$
\delta_{k, n}=\left[\frac{k-n \bmod k}{k+[(n-1) / k]}\right], \quad n \geq 1 .
$$

These e.g. functions will be used throughout the remainder of this paper.

It should be noted that e.g. functions are closed under addition, multiplication, and composition. Moreover, if $f(n)$ and $g(n)$ represent two e.g. sequences, the "shuffled" sequence

$$
h(n)= \begin{cases}f((n+1) / 2) & n \text { odd } \\ g(n / 2) & n \text { even }\end{cases}
$$

is also e.g., for

$$
\begin{gathered}
h(n)=c(n) f((n+1) / 2)+d(n) g(n / 2), \\
\text { where } c(n)=[(n+1) / 2]-[n / 2] \text {, and } d(n)=[(n+2) / 2]-[(n+1) / 2] .
\end{gathered}
$$

\section{FINITE SEQUENCES OF POSITIVE INTEGERS.}

We now show

Proposition 1. Every finite increasing (injective) sequence of positive integers, and its complement, are e.g.

(i) If $x_{1}<x_{2}<\ldots<x_{n}$, finding an e.g. function $f$ generating the $x_{i}{ }^{\prime} s$ is similar to constructing an interpolating polynomial (see [5], for example) through the $n$ points $\left(1, x_{1}\right), \ldots,\left(n, x_{n}\right)$. If for each $i=1,2, \ldots, n$, we define $g_{j}(x)=(x-1)(x-2) \ldots(x-i+1)(x-i-1) \ldots(x-n)$, so that $g_{j}(k)=0$ when $i \neq k$, then 
the e.g. function

$$
f(x)=\frac{x_{1}}{g_{1}(1)} g_{1}(x)+\ldots+\frac{x_{n}}{g_{n}(n)} g_{n}(x)
$$

satisfies $f(i)=x_{i}$. Alternatively, one can use Kronecker delta functions.

(ii) The complement of the sequence $\left\langle x_{1}, x_{2}, \ldots, x_{n}\right\rangle$ is also e.g. The set $\left\{m: m<x_{n}, m \neq x_{1}, x_{2}, \ldots, x_{n}\right\}$ in increasing order represents a finite sequence; hence there is an e.g. function generating $i t$, by (i). Suppose this set has cardinality $t$. We need an e.g. function $h$ for which

$$
h(i)= \begin{cases}f(i) & i \leq t ; \\ x_{n}+(i-m) & i>t .\end{cases}
$$

If we define

$$
h(i)=\delta_{1, i} f(1)+\ldots+\delta_{t, 1} f(t)+\left(1-\delta_{1, i}-\ldots-\delta_{t, 1}\right)\left(x_{n}+1-m\right),
$$

then $h$ satisfies (2.1).

\section{COMPLEMENTS OF POL YMOMIAL SEQUENCES.}

As mentioned in the introduction, the authors of [4] derive formula (1.2), and thus establish that the complement of the sequence $\left\langle x^{n}\right\rangle$ is e.g. for every positive integer $\mathrm{n}$. They ask whether indeed the complement of every increasing e.g. sequence is again e.g. This question remains open, but our following improvement on their main result induces a guess that the answer may be 'yes':

Conjecture 1. Let $f(x)$ be a polynomial of positive degree in the indeterminate $x$, and each of whose coefficients is a non-negative integer. Then of course the sequence $\langle f(i)\rangle$ is strictly increasing and e.g. The complement of this sequence is also e.g.

In order to establish the second conclusion in Conjecture 1, we first obtain some preliminary results. In fairness to the reader, we state the above result as a conjecture rather than a theorem.

(i) Complements of $a x^{n} ; n, a>0$.

Let $f_{(n, a)}(i)=$ the ${ }^{i}$ th positive integer which is not of the form $a x^{n}$, for some fixed positive integers $n$ and $a,(n, a) \neq(1,1)$. For $a=1$ and $n$ at least 2 , $f_{(n, a)}$ is precisely the function $f_{n}$ given by $(1.2)$. We define for integers $i>0$

$$
\begin{aligned}
& z_{(n, a)}(i)=\left[(1 / a)^{1 / n}\right] \equiv z ; \quad \text { and } \\
& p_{(n, a)}(i)=a(z+1)^{n}-z \equiv p .
\end{aligned}
$$

Then we may prove

Theorem 1. $f_{(n, a)}(i)=i+z(n, a)(i)+[i / p(n, a)(i)]$. 
The proof of Theorem 1 is long and is similar to the proof of the Theorem found in [4]. The approach of the current author consisted of establishing that exactly $k$ integers from the set $\left\{1,2,3, \ldots, a k^{n}+k\right\}$ are omitted from the sequence $\langle f(n, a)(i)\rangle$; that is, $f_{(n, a)}\left(a k^{n}\right)=a k^{n}+k$. (See the Lemma in [4].) Next, if the sequence skips an integer, then that integer is of the form $a x^{n}$; that is, the $\left(a k^{n}-k\right)$ th term < $a k^{n}$, the $\left(a k^{n}-k+1\right)$ th term $>a k^{n}$, and the $\left(a k^{n}\right)$ th term $=a k^{n}+k$. Finally, the sequence is shown to be increasing.

Computer-oriented readers will find that confirming the accuracy of Theorem 1, and subsequent results, for integers less than, say, 1 million, is both instructive and rewarding.

As an illustration of Theorem 1, consider the polynomial $5 x^{4}$. Then $f(4,5)$ generates the complement of the sequence $\langle 5,80,405, \ldots\rangle$. The 100 th term of the complement is 102. Indeed, $z_{(4,5)}(100)=\left[(100 / 5)^{1 / 4}\right]=2 ; p_{(4,5)}(100)=5(2+1)^{4}$ $-2=403$; and $f_{(4,5)}(100)=100+2+[100 / 403]=100+2+0=102$.

The Theorem in [4] comes as a corollary to Theorem 1.

(ii) Complements of $a x^{n}+b ; a, n, b>0$.

Let $g_{(n, a, b)}(i)$ be the $i$ th term of the complement of the polynomial sequence $<a x^{n}+b>$. Since $x>0, g(n, a, b)(i)=i$ for $i \leq b$. Then, with the result of Theorem 1, for $i>b, g(n, a, b)(i)$ is the sum of $b$ and an element of the complement defined by $\left\langle a x^{n}\right\rangle$. Explicitly, we have

Theorem 2. $g_{(n, a, b)}(i)=\delta_{1, i}(1)+\delta_{2, i}(2)+\ldots+\delta_{b, j}(b)+$ $\left(1-\delta_{1, i}-\ldots-\delta_{b, i}\right)(f(n, a)(i-b)+b)$.

For example, the 106th term of the complement of $\left\langle 5 x^{4}+6\right\rangle$ is $108=102+6=$ $f_{(4,5)}(100)+6=f_{(4,5)}(106-6)+6$.

Likewise, the terms of the complement of $\left\langle a x^{n}+b x^{m}>, n>m\right.$, are "systematic" sums of a term of $\left\langle b x^{m}\right\rangle$ and a term of the complement of $\left\langle a x^{n}\right\rangle$. Inductive arguments then show that complements of sequences generated by polynomials of positive degree with non-negative integer coefficients are e.g. These proofs are omitted. They are involved and their details must be left to the interested reader.

As an example, however, let $p(x)=2 x^{2}+3 x$. The sequence $\langle 3 x\rangle$ is equal to $\langle 3,6,9,12,15,18,21, \ldots\rangle$, and the complement of the sequence $\left\langle 2 x^{2}\right\rangle$ is $r=$ $\langle 1,3,4,5,6,7,9,10,11, \ldots, 15,16,17,19,20, \ldots, 29,30,31,33, \ldots\rangle$. The first four terms of the complement $t$ of $\left\langle 2 x^{2}+3 x\right\rangle$ are $1,2,3$, and 4 . Subsequent terms are generated by the following chart. 


\begin{tabular}{|c|c|c|c|c|c|}
\hline$i$ & $i$ th term of $\mathbf{t}$ & $=$ & term of $\langle 3 x\rangle$ & + & term of $r$ \\
\hline 5 & 6 & $=$ & 3 & + & 3 \\
\hline 6 & 7 & $=$ & 3 & + & 4 \\
\hline 7 & 8 & $=$ & 3 & + & 5 \\
\hline 8 & 9 & $=$ & 3 & + & 6 \\
\hline 9 & 10 & $=$ & 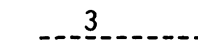 & \pm & 7 \\
\hline 10 & 11 & $=$ & 6 & + & 5 \\
\hline 11 & 12 & $=$ & 6 & + & 6 \\
\hline 12 & 13 & $=$ & 6 & + & 7 \\
\hline 13 & 15 & $=$ & 6 & + & 9 \\
\hline$\cdot$ & $\cdot$ & • & $\cdot$ & • & $\cdot$ \\
\hline$\dot{.}$ & $\dot{.}$ & $\dot{.}$ & . & $\dot{.}$ & $\dot{.}$ \\
\hline 21 & 23 & $=$ & $\underline{6}$ & \pm & -17 \\
\hline 22 & 24 & $=$ & 9 & + & 15 \\
\hline 23 & 25 & $=$ & 9 & + & 16 \\
\hline 24 & 26 & $=$ & 9 & + & 17 \\
\hline 25 & 28 & $=$ & 9 & + & 19 \\
\hline$\cdot$ & $\cdot$ & $\cdot$ & $\cdot$ & $\cdot$ & - \\
\hline$\dot{.}$ & $\dot{.}$ & $\dot{.}$ & $\dot{.}$ & $\dot{.}$ & $\dot{.}$ \\
\hline 37 & 40 & $=$ & 9 & \pm & 31 \\
\hline 38 & 41 & $=$ & 12 & + & 29 \\
\hline$\cdot$ & - & • & - & $\cdot$ & - \\
\hline$\cdot$ & - & - & $\cdot$ & $\cdot$ & • \\
\hline
\end{tabular}

We leave as a problem the following question posed in [4].

Open Problem 1. Is the complement of an e.g. sequence again e.g.?

Perhaps a non-e.g. sequence may be described as a "discrete transcendental" function. As mentioned in the introduction, there are uncountably many of these. The existence of a non-e.g. sequence may be shown, nonconstructibly, with the Axiom of Choice. Let $\left\{A_{i}\right\}$ be a countable collection of mutually disjoint sets of positive integers. Use the Axiom to define a function $h$ on $Z^{+}$such that $h(i) \varepsilon$ $A_{i}, i=1,2, \ldots$. These "choice" functions cannot be exhibited.

Open Problem 2. Exhibit a non-e.g. sequence of positive integers.

It is a conjecture that the sequence of prime numbers and the sequence of composite numbers are non-e.g. The partition function (see [6], Chap. 10) may also be non-e.g.

Finally, is there a connection between non-e.g. functions and sequences related to the decimal expansions of transcendental real numbers in the unit interval $[0,1]$ ? Define an equivalence relation $\equiv$ on the set of all sequences via $\left\langle a_{n}\right\rangle \equiv\left\langle b_{n}\right\rangle$ if the base-ten decimal numeral formed by juxtaposing (or concatenating) 
the terms of $\left\langle a_{n}\right\rangle$ is equal to that formed from $\left\langle b_{n}\right\rangle$; in other words, if $0 . a_{1} a_{2} \ldots$ $=0 . b_{1} b_{2} \ldots$. For example, the sequence $\langle 1,2,3,4, \ldots\rangle$ is equivalent to the sequence $<12,34,56, \ldots\rangle$. Which equivalence classes define irrational numbers? Which ones define transcendental numbers?

4. RATIONAL, IRRATIONAL, AND TRANSCENDENTAL REPRESENTATIONS.

Every rational number has an e.g. representation. For example, consider the rational decimal $r=0.717171 \ldots$. Then

$$
\begin{aligned}
r & =.71+.0071+.000071+\ldots \\
& =\frac{71}{10^{2}}+\frac{71}{10^{4}}+\frac{71}{10^{6}}+\ldots \\
& =\frac{71}{10^{2}} \sum_{i=0}^{\infty}\left(\frac{1}{10^{2}}\right)^{i} .
\end{aligned}
$$

The geometric series (4.1) is convergent, and is the limit of its partial sums

$$
s_{n}=\frac{71}{10^{2}} \sum_{i=0}^{n}\left(\frac{1}{10^{2}}\right)^{1}=\frac{71}{10^{2}-1}\left(1-10^{-2(n+1)}\right) .
$$

If we now define for $n \geq 1$,

$$
a_{n}=10^{2 n} s_{n-1}=\frac{71}{10^{2}-1}\left(10^{2 n}-1\right),
$$

then $a_{1}=71, a_{2}=7171, a_{3}=717171$, etc. Thus $r$ is the decimal formed by juxtaposition of the terms of $\left\langle a_{n}\right\rangle$.

In general, let $x=0 . a b$ be a rational decimal between 0 and 1 , where $a$ is the non-repeating part of $x$, and $b$ is the repeating part of $x$, comprised of $t$ digits. (For example, if $x=0.56 \overline{892}$, then $a=56, b=892, t=3$.) Then $x$ may be formed by juxtaposing or concatenating the terms of the e.g. sequence

$$
f(n)=\delta_{1, n^{a}}+\left(1-\delta_{1, n}\right) \cdot \frac{b}{10^{t}-1}\left(10^{t(n-1)}-1\right) .
$$

Thus we have

Proposition 2. Every rational decimal has an e.g. representation; in particular, it is exponentially generated.

It is not the case, however, that every exponentially generated sequence gives rise to a rational number, or even an algebraic number. For instance, the number generated by $\left\langle 10^{-j !}\right\rangle$ is transcendental! (See [7, p. 92].)

Many irrational decimals, however, are e.g. For example, $f(n)=n$ gives rise 
to the irrational $0.1234 \ldots$. The irrational number $0.121122111222 \ldots$ can be formed by "shuffling" the terms of the sequence $\langle 1,11,111, \ldots\rangle$ with those of $\langle 2,22$, $222, \ldots>$. Both of these sequences, and hence their shuffled sequence, are e.g. Finally, every real decimal has a non-e.g. representation by the Axiom of Choice, but uncountably many have no e.g. representation. This prompts the following open question.

Open Problem 3. Is a real decimal which has no e.g. representation necessarily transcendental?

ACKNOWLEDGEMENT. The author wishes to acknowledge James Chapman for his assistance in this project. He is also grateful to the referee for the valuable comments which led to the present appearance of this paper.

\section{REFERENCES}

1. HONSBERGER, R. Ingenuity in Mathematics, Mathematical Association of America, 1970.

2. HALBERSTAM, H. In Praise of Arithmetic, in The Teaching of Algebra at the PreCollege Level edited by P. Braunfield and W. E. Deskins, CEMREL, St. Louis, 1975.

3. NELSON, R.D. Sequences which Omit Powers, Math. Gazette 72 (1988), 208-211.

4. DOS REIS, A.J. and SILBERGER, D.M. Generating Nonpowers by Formula, Math. Mag. 63 (1990), 53-55.

5. CHENEY, W. and KINCAID, D. Numerical Mathematics and Computing, Brooks/Cole, Monterey, CA, 1980 .

6. NIVEN, I. and ZUCKERMAN, H.S. An Introduction to the Theory of Numbers, John Wiley, New York, 1980.

7. NIVEN, I. Irrational Numbers, Carus Mathematical Monograph Number 11, Mathematical Association of America, 1956. 


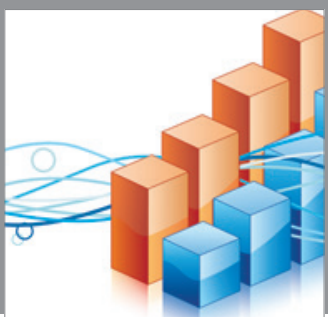

Advances in

Operations Research

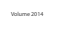

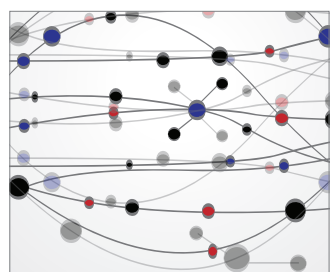

\section{The Scientific} World Journal
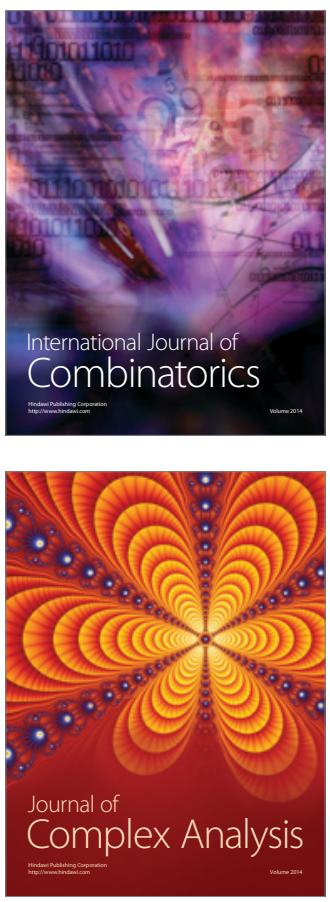

International Journal of

Mathematics and

Mathematical

Sciences
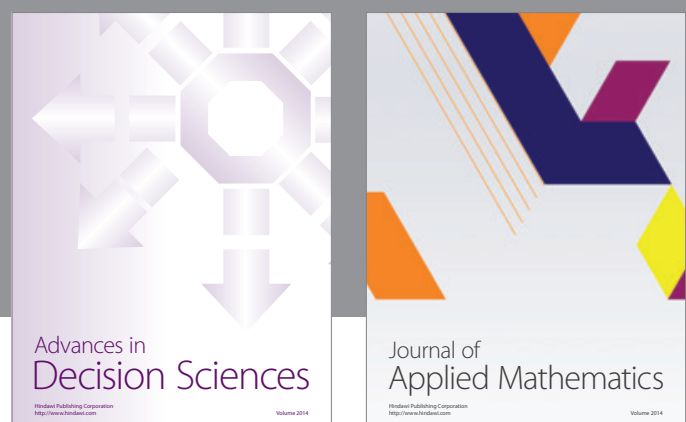

Journal of

Applied Mathematics
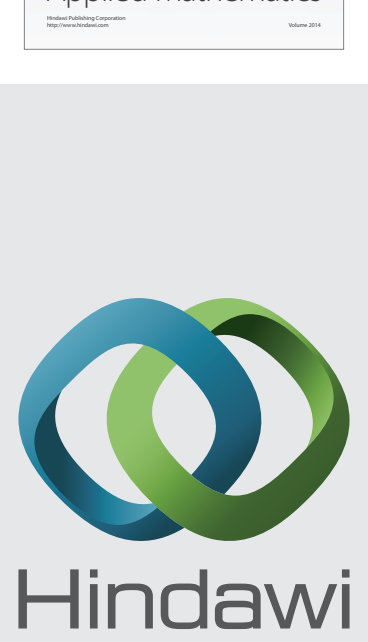

Submit your manuscripts at http://www.hindawi.com
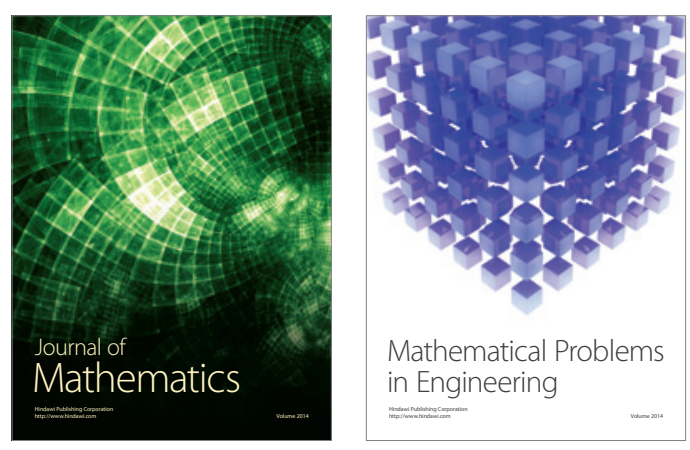

Mathematical Problems in Engineering
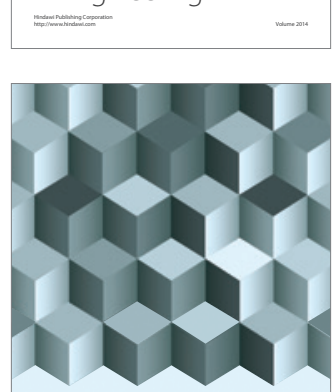

Journal of

Function Spaces
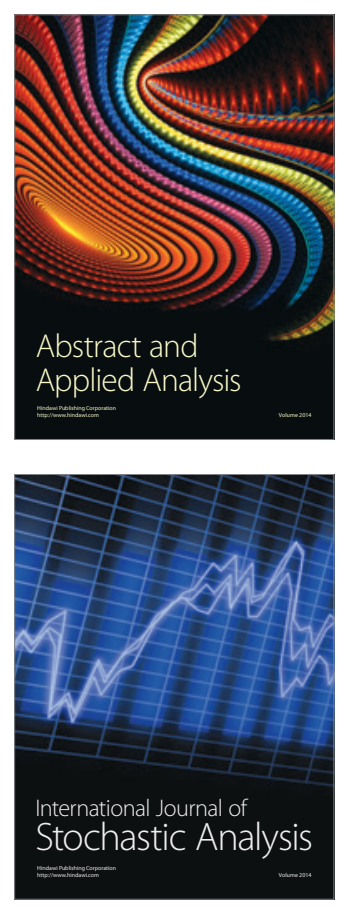

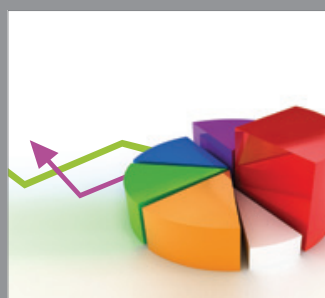

ournal of

Probability and Statistics

Promensencen
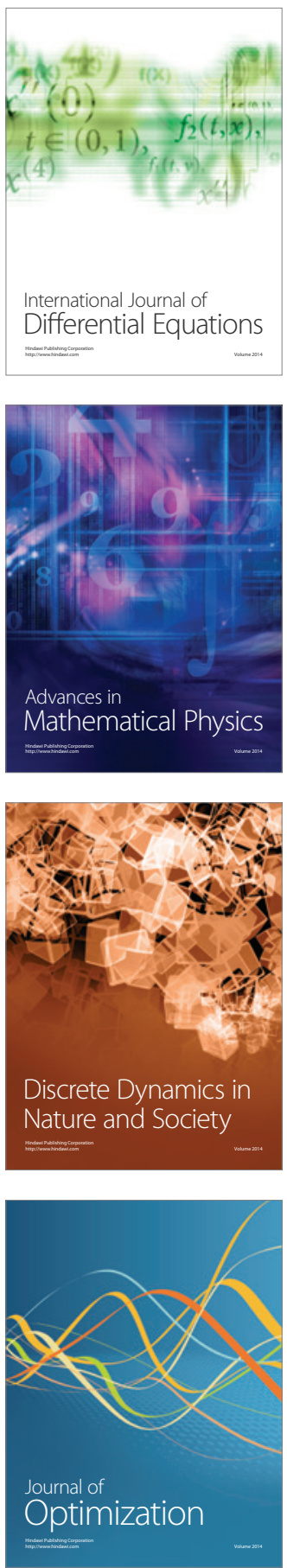